\title{
Extended point values of distributions
}

\author{
Ricardo Estrada* \\ Department of Mathematics, Louisiana State University, Baton Rouge, LA 70803, USA
}

(Received: 17 October 2020. Received in revised form: 15 December 2020. Accepted: 15 December 2020. Published online: 19 December 2020.)

(c) 2020 the author. This is an open access article under the CC BY (International 4.0) license (www. creativecommons.org/licenses/by/4.0/).

\begin{abstract}
An extension method for linear functionals is given. The proposed method provides extensions of a linear functional $T$ defined on a subspace $X$ of a vector space $Y$ over a field $K$, by using a suitable isomorphism $S: Y \longrightarrow Y$ that satisfies $S(X)=X$ and $T S=T$. The extension $T_{\text {ext }}: X_{\text {ext }} \longrightarrow K$ is linear, and it is defined over a vector space $X_{\text {ext }}$ that contains $X$. Several illustrations are considered, including symmetric values, extension with respect to dilations, extended Cesàro summability of series, and extended multidimensional point values.
\end{abstract}

Keywords: distributional point values; summability.

2020 Mathematics Subject Classification: $46 \mathrm{~F} 10$

\section{Introduction}

It is many times convenient to assign a finite value to a divergent limit, such as the limits of partial sums of divergent series or the limit of integrals over finite intervals as these intervals become infinite. Using divergent series and integrals to find the answer to several problems, in particular the result of convergent processes, is a very old and fruitful practice as can be seen in Hardy's book [8] or in Bromwich's book [1]. A great variety of summability methods have been employed throughout the years. Distributional theory often employs other methods, regularization methods, as for instance Hadamard finite part limits [7], of importance in the area of partial differential equations, since they provide fundamental solutions. Finite part regularized values are also useful in number theory [3]. The study of divergent limits has a also close relationship with pseudofunctions $[9,16]$. Paycha [12] explains the need to employ regularized values for divergent series and integrals in other areas as noncommutative geometry and quantum mechanics.

In a recent article, Sasane [15] introduced an extension procedure to assign a point value to distributions that do not have such value, and employed this to find extended values for divergent series. His method was motivated by a trick used by Ramanujan to assign the value $-1 / 12$ to the divergent series $\sum_{n=1}^{\infty} n$, a series that, interestingly, gives the Casimir effect, the value of the attractive force between two parallel perfect conductor plates in vacuum. Sasane's method has several shortcomings, since in general it is not linear, nor is defined in a vector space. In fact, even though $\sum_{n=1}^{\infty} n^{2}$ also has an extended value, the series $\sum_{n=1}^{\infty}\left(n+n^{2}\right)$ does not.

The purpose of this article is to give an extension method of which a general form of Sasane's extension is the first step. Our method provides extensions of a linear functional $T$ defined on a subspace $X$ of a vector space $Y$ over a field $K$, by using a suitable isomorphism $S: Y \longrightarrow Y$ that satisfies $S(X)=X$ and $T S=T$. The extension $T_{\text {ext }}: X_{\text {ext }} \longrightarrow K$ is linear, and it is defined over a vector space $X_{\text {ext }}$ that contains $X$. We provide several illustrations by considering different spaces and operators that satisfy our conditions, including symmetric values, extension with respect to dilations, extended Cesàro summability of series, and extended multidimensional point values.

\section{Preliminaries}

We refer to the texts for the basic ideas about distributions $[9,16]$. Notions from the local analysis of distributions can be found in $[4,6,13,14,17]$. Distributional point values were first defined by Łojasiewicz [10,11], who defined the value of a distribution $f \in \mathcal{D}^{\prime}\left(\mathbb{R}^{n}\right)$ at the point $\mathbf{x}_{0}$ as the limit

$$
f\left(\mathbf{x}_{0}\right)=\lim _{\varepsilon \rightarrow 0} f\left(\mathbf{x}_{0}+\varepsilon \mathbf{x}\right),
$$

\footnotetext{
*E-mail address: restrada@math.lsu.edu
} 
if the limit exists in $\mathcal{D}^{\prime}\left(\mathbb{R}^{n}\right)$, that is, $f\left(\mathbf{x}_{0}\right)=\gamma$ if

$$
\lim _{\varepsilon \rightarrow 0}\left\langle f\left(\mathbf{x}_{0}+\varepsilon \mathbf{x}\right), \phi(\mathbf{x})\right\rangle=\gamma \int_{\mathbb{R}^{n}} \phi(\mathbf{x}) \mathrm{d} \mathbf{x},
$$

for all test functions $\phi \in \mathcal{D}\left(\mathbb{R}^{n}\right)$.

Distributional point values are already extended values since if a distribution is given by a continuous function in a neighborhood of $\mathrm{x}_{0}$ then the distributional value at this point is the ordinary value. They are a sort of average value, actually a Cesàro type average [4]. In one dimension they allow one to extend the Lebesgue integral to the distributional integral [5].

References to summability methods are [1,8] and, from a distributional approach, [4]. Ideas on Cesàro summability of series can be seen in those references. Interestingly, point values of periodic distributions and the Cesàro sum of its Fourier series are very closely related [2] as we explain in Section 7.

\section{A general extension procedure}

Let $Y$ be a vector space over a field $K$, let $X$ be a linear subspace and let

$$
T: X \longrightarrow K
$$

be a linear map. Let us also suppose that

$$
S: Y \longrightarrow Y
$$

is a linear isomorphism.

Definition 3.1. We say that $T$ is invariant with respect to $S$ and $X$ if the following two conditions are satisfied:

1. $S \varphi \in X$ if and only if $\varphi \in X$, that is, $S(X)=X$;

2. $T S(\varphi)=T(\varphi)$ for all $\varphi \in X$.

If $T$ is invariant with respect to $S$ then we can define an extension of $T$ in the following fashion.

Definition 3.2. The set $X_{\text {ext }}^{\{1\}}$ is formed by those elements $\varphi \in Y$ for which there exists $\kappa \in K, \kappa \neq 1$, such that

$$
\varphi_{\kappa}=\varphi-\kappa S \varphi \in X
$$

It would be convenient to denote as $X_{\kappa}$ the set of $\varphi$ for which $\varphi_{\kappa} \in X$, so that

$$
X_{\mathrm{ext}}^{\{1\}}=\bigcup_{\kappa \neq 1} X_{\kappa} .
$$

Naturally $X \subset X_{\kappa}$ for all $\kappa$, but if $\varphi \in X_{\text {ext }}^{\{1\}} \backslash X$ then the constant $\kappa$ is unique, as the next lemma shows.

Lemma 3.1. Let $\varphi \in X_{\text {ext }}^{\{1\}}$. If $\kappa \neq \lambda$ are scalars, then $\varphi_{\kappa}$ and $\varphi_{\lambda}$ both belong to $X$ if and only if $\varphi \in X$. If $\kappa \neq \lambda$ then $X_{\kappa} \cap X_{\lambda}=X$.

Proof. If $\varphi_{\kappa}$ and $\varphi_{\lambda}$ both belong to $X$ for two different scalars $\kappa$ and $\lambda$, then it follows that

$$
S \varphi=(\kappa-\lambda)^{-1}\left(\varphi_{\kappa}-\varphi_{\lambda}\right)
$$

also belongs to the vector subspace $X$, and consequently $\varphi \in X$. Conversely, if $\varphi \in X$ then so does $S \varphi$ and thus $\varphi_{\kappa} \in X$ for any $\kappa \in K$.

Actually, a little more is true. If $\varphi \in X_{\text {ext }}^{\{1\}} \backslash X$ let us denote by $\kappa(\varphi)$ the unique $\kappa$ such that $\varphi \in X_{\kappa}$.

Lemma 3.2. The constant $\kappa$ depends only on the equivalence class of $\varphi$ in the quotient space $Y / X,[\varphi] \in Y / X$, that is $\kappa=\kappa([\varphi])$ for $[\varphi] \neq[0]$.

Proof. Indeed, if $\varphi \in X_{\mathrm{ext}}^{\{1\}} \backslash X$ and $[\varphi]=[\psi]$ then $\varphi-\psi=\chi \in X$ and consequently if $\kappa=\kappa(\varphi)$, that is, $\varphi \in X_{\kappa}$, then $\psi_{\kappa}=\varphi_{\kappa}-\chi_{\kappa} \in X$, so that $\psi \in X_{\kappa}$, hence $\kappa=\kappa(\psi)$. 
We observe also that if $\varphi \in X_{\text {ext }}^{\{1\}} \backslash X$ then

$$
\kappa(\lambda \varphi)=\kappa(\varphi), \text { for all } \lambda \in K
$$

We can now define an extension operator

$$
T_{\mathrm{ext}}^{\{1\}}: X_{\mathrm{ext}}^{\{1\}} \longrightarrow K
$$

as follows.

Definition 3.3. If $\varphi \in X_{e x t}^{\{1\}}$ we put

$$
T_{e x t}^{\{1\}}(\varphi)=(1-\kappa)^{-1} T\left(\varphi_{\kappa}\right)
$$

if $\varphi \in X_{\kappa}$.

Notice that if $\varphi \in X$ then $\varphi \in X_{\kappa}$ for any $\kappa \neq 1$, but $T_{\text {ext }}^{\{1\}}(\varphi)$ is well defined because $(1-\kappa)^{-1} T\left(\varphi_{\kappa}\right)=T(\varphi)$ for any $\kappa$. Notice also that the restriction $\kappa \neq 1$ that we have imposed is needed for (2) to make sense.

The space $X_{\text {ext }}^{\{1\}}$ will not be a vector space and the function $T_{\text {ext }}^{\{1\}}$ will not be linear, in general. In fact, we have the following result.

Lemma 3.3. If $\phi, \psi$, and $\phi+\psi$ all belong to $X_{\text {ext }}^{\{1\}} \backslash X$ then

$$
\kappa(\varphi)=\kappa(\psi)=\kappa(\varphi+\psi) .
$$

Proof. Let $\lambda=\kappa(\varphi), \omega=\kappa(\psi)$, and $\mu=\kappa(\varphi+\psi)$ and suppose $\lambda \neq \omega$. Then $\lambda \neq \mu$ or $\omega \neq \mu$; let us say the first one holds, to fix the ideas. We have that $\varphi-\lambda S \varphi, \psi-\omega S \psi$, and $(\varphi+\psi)-\mu S(\varphi+\psi)$ all belong to $X$. Since $X$ is a vector space it follows by adding the first two and subtracting the third that

$$
(\mu-\lambda) S \varphi+(\mu-\omega) S \psi \in X,
$$

and consequently

$$
(\mu-\lambda) \varphi+(\mu-\omega) \psi \in X
$$

Hence

$$
[\varphi]=\left[\left(\frac{\mu-\omega}{\lambda-\mu}\right) \psi\right],
$$

which in view of the Lemma 3.2 and (1) yields $\lambda=\kappa(\varphi)=\kappa(\psi)=\omega$, a contradiction.

For each $\kappa \neq 1$ the space $X_{\kappa}$ is a vector space. However, as the Lemma 3.3 shows, if $\lambda \neq \mu$ and $\varphi \in X_{\lambda} \backslash X, \psi \in X_{\omega} \backslash X$ then $\varphi+\psi \notin X_{\text {ext }}^{\{1\}}$. Therefore, unless $X_{\text {ext }}^{\{1\}}=X$ or $X_{\text {ext }}^{\{1\}}=X_{\kappa_{0}}$ for a single value $\kappa_{0}$ with $X_{\kappa_{0}} \neq X$, the space $X_{\text {ext }}^{\{1\}}$ is not a vector space. In order to construct a linear extension of $T$, defined in a vector space, we need to go beyond $T_{\mathrm{ext}}^{\{1\}}$ and $X_{\mathrm{ext}}^{\{1\}}$, as we explain in the next section.

\section{Further extensions}

The method of the previous section can be applied whenever $T$ is invariant with respect to $S$ and $X$. Therefore we can apply it again to the space $X_{\kappa}, \kappa \neq 1$, and the operator

$$
T_{k}=\left.T_{\mathrm{ext}}^{\{1\}}\right|_{X_{\kappa}}
$$

because of the next observation.

Lemma 4.1. The operator $T_{k}$ is invariant with respect to $S$ and $X_{\kappa}$.

Proof. Notice that $\varphi \in X_{\kappa}$ if and only if $\varphi-\kappa S \varphi \in X$, and since $T$ is invariant with respect to $S$ and $X$, this is equivalent to $S \varphi-\kappa S(S \varphi) \in X$, that is $S \varphi \in X_{\kappa}$.

The operator $T_{\kappa}$ is clearly linear. Also, if $\varphi \in X_{\kappa}$,

$$
\begin{aligned}
T_{\kappa} S(\varphi) & =T_{\mathrm{ext}}^{\{1\}}(S \varphi)=(1-\kappa)^{-1} T\left(S \varphi_{\kappa}\right) \\
& =(1-\kappa)^{-1} T\left(\varphi_{\kappa}\right)=T_{\mathrm{ext}}^{\{1\}}(\varphi)=T_{\kappa}(\varphi),
\end{aligned}
$$

namely, $T_{\kappa} S=T_{\kappa}$, as required. 
If we now start from $X_{\kappa}$ we can construct spaces $\left(X_{\kappa}\right)_{\lambda}$ for any $\lambda \neq 1$. Actually the same space is obtained if we start from the space $X_{\lambda}$.

Lemma 4.2. If $\kappa \neq 1$ and $\lambda \neq 1$ then

$$
\left(X_{\kappa}\right)_{\lambda}=\left(X_{\lambda}\right)_{\kappa}
$$

Proof. Indeed, $\varphi \in\left(X_{\kappa}\right)_{\lambda}$ if and only if $\left(\varphi_{\kappa}\right)_{\lambda} \in X$, and

$$
\left(\varphi_{\kappa}\right)_{\lambda}=\varphi_{\kappa}-\lambda S \varphi_{\kappa}=\varphi-(\kappa+\lambda) S \varphi+\kappa \lambda S^{2} \varphi=\left(\varphi_{\lambda}\right)_{\kappa}
$$

From now on we shall denote as $\varphi_{\kappa, \lambda}$ the element $\left(\varphi_{\kappa}\right)_{\lambda}=\left(\varphi_{\lambda}\right)_{\kappa}$ and the space $\left(X_{\kappa}\right)_{\lambda}=\left(X_{\lambda}\right)_{\kappa}$ as $X_{\kappa, \lambda}$. We then construct an extension of $T$ from $X$ to $X_{\kappa, \lambda}$ as

$$
T_{\kappa, \lambda}(\varphi)=\frac{T\left(\varphi_{\kappa, \lambda}\right)}{(1-\kappa)(1-\lambda)}=\frac{T\left(\varphi_{\kappa, \lambda}\right)}{1-\kappa-\lambda+\kappa \lambda} .
$$

The space $X_{\kappa, \lambda}$ is a vector space and $T_{\kappa, \lambda}: X_{\kappa, \lambda} \rightarrow K$ is linear. On the other hand, the set

$$
X_{\mathrm{ext}}^{\{2\}}=\bigcup_{\kappa \neq 1, \lambda \neq 1} X_{\kappa, \lambda}
$$

will not be a vector space, in general, and the operator $T_{\text {ext }}^{\{2\}}$ from $X_{\text {ext }}^{\{2\}}$ to $K$ given by $T_{\text {ext }}^{\{2\}}(\varphi)=T_{\kappa, \lambda}(\varphi)$ if $\varphi \in X_{\kappa, \lambda}$ will not be linear, in general. Notice, however, that if $\varphi \in X_{\kappa}, \psi \in X_{\lambda}$ then $\varphi+\psi$, which in general does not belong to $X_{\text {ext }}^{\{1\}}$, will belong to $X_{\text {ext }}^{\{2\}}$ and actually

$$
T_{\mathrm{ext}}^{\{2\}}(\varphi+\psi)=T_{\mathrm{ext}}^{\{2\}}(\varphi)+T_{\mathrm{ext}}^{\{2\}}(\psi) .
$$

More generally for $\left(\kappa_{1}, \ldots, \kappa_{n}\right) \in K^{n}$, with $\kappa_{j} \neq 1$ for all $j$, we may define the vector spaces $X_{\kappa_{1}, \ldots, \kappa_{n}}$ recursively as

$$
X_{\kappa_{1}, \ldots, \kappa_{n}}=\left(X_{\kappa_{1}, \ldots, \kappa_{n-1}}\right)_{\kappa_{n}}
$$

We can also define extension operators from $X_{\kappa_{1}, \ldots, \kappa_{n}}$ to $K$ as

$$
T_{\kappa_{1}, \ldots, \kappa_{n}}=\left(T_{\kappa_{1}, \ldots, \kappa_{n-1}}\right)_{\kappa_{n}} .
$$

The set

$$
X_{\mathrm{ext}}^{\{n\}}=\bigcup_{\kappa_{j} \neq 1} X_{\kappa_{1}, \ldots, \kappa_{n}},
$$

will not be a vector space, in general, and the operator $T_{\operatorname{ext}}^{\{n\}}$ from $X_{\text {ext }}^{\{n\}}$ to $K$ given by $T_{\operatorname{ext}}^{\{n\}}(\varphi)=T_{\kappa_{1}, \ldots, \kappa_{n}}(\varphi)$ if $\varphi \in X_{\kappa_{1}, \ldots, \kappa_{n}}$ will not be linear, in general. However, we have the ensuing extension result.

Theorem 4.1. The set

$$
X_{\text {ext }}=\bigcup_{n=1}^{\infty} X_{\text {ext }}^{\{n\}}
$$

is a vector subspace of $Y$. The operator

$$
T_{\text {ext }}: X_{\text {ext }} \rightarrow K
$$

given by

$$
T_{\text {ext }}(\varphi)=T_{\text {ext }}^{\{n\}}(\varphi)=T_{\kappa_{1}, \ldots, \kappa_{n}}(\varphi),
$$

if $\varphi \in X_{\kappa_{1}, \ldots, \kappa_{n}} \subset X_{\text {ext }}^{\{n\}}$ is a linear operator that extends $T: X \rightarrow K$. Furthermore,

$$
T_{\text {ext }}(S \varphi)=T_{\text {ext }}(\varphi)
$$

Proof. We just need to notice that

$$
X_{\mathrm{ext}}=\bigcup_{n=1}^{\infty} \bigcup_{\kappa_{j} \neq 1} X_{\kappa_{1}, \ldots, \kappa_{n}},
$$

is a union of vector subspaces of $Y$ and that the operators $T_{\kappa_{1}, \ldots, \kappa_{n}}$ are linear, extend $T$, and satisfy the equation corresponding to (3). 
We can also give an alternative formula for $T_{\text {ext }}$. Let $\varphi \in X_{\text {ext }}$, say $\varphi \in X_{\kappa_{1}, \ldots, \kappa_{n}}$, where $\kappa_{j} \neq 1$ for all $j$. Let $p=p_{\kappa_{1}, \ldots, \kappa_{n}}$ be the polynomial

$$
p(x)=\left(1-\kappa_{1} x\right) \cdots\left(1-\kappa_{n} x\right)
$$

Then

$$
\varphi_{\kappa_{1}, \ldots, \kappa_{n}}=p(S)(\varphi) \in X
$$

and

$$
T_{\text {ext }}(\varphi)=\frac{T\left(\varphi_{\kappa_{1}, \ldots, \kappa_{n}}\right)}{p(1)} .
$$

Notice that when the field $K$ is algebraically closed then a polynomial $p$ is of the form (4) if and only if $p(0)=1$ and $p(1) \neq 0$.

It may be useful to use another notation for the extension operator, namely, if $T_{\text {ext }}(\varphi)=\gamma$ we put

$$
T(\varphi)=\gamma \quad(\text { ext, } S) .
$$

We will consider several illustrations of the extension procedure in the next sections. Naturally the method applies to any operator, but we concentrate on extended point values.

\section{Symmetric values}

In our first illustration we consider the one dimensional case and take

$$
\begin{gathered}
Y=\mathcal{D}^{\prime}(\mathbb{R}), \\
X=\{f \in Y: \text { the distributional point value } f(0) \text { exists }\},
\end{gathered}
$$

and $T$ is that distributional value,

$$
T(f)=f(0) .
$$

Let now $S$ be the operator

$$
S(f)(x)=f(-x) .
$$

Clearly $T$ is invariant with respect to $S$ and $X$. We shall see that in this case

$$
X_{\kappa}=X, \quad \kappa \neq-1,
$$

while

$$
X_{-1} \supsetneq X
$$

In fact, if we decompose $f$ in its even and odd parts,

$$
f=f_{\text {even }}+f_{\text {odd }},
$$

then

$$
X_{-1}=\left\{f \in Y: \text { the distributional point value } f_{\text {even }}(0) \text { exists }\right\} .
$$

Indeed, if $\kappa \neq-1$ and $f_{\kappa} \in X, f_{\kappa}(0)=L_{\kappa}$ distributionally, then also the distributional point values $\left(f_{\kappa}\right)_{\text {even }}(0)=\left(f_{\text {even }}\right)_{\kappa}(0)$ exist and equal $L_{\kappa}$ while $\left(f_{\kappa}\right)_{\text {odd }}(0)=\left(f_{\text {odd }}\right)_{\kappa}(0)$ exist and equal 0 . But

$$
\begin{gathered}
\left(f_{\text {even }}\right)_{\kappa}(0)=f_{\text {even }}(x)-\left.\kappa f_{\text {even }}(-x)\right|_{x=0}=\left.(1-\kappa) f_{\text {even }}(x)\right|_{x=0}, \\
\left(f_{\text {odd }}\right)_{\kappa}(0)=f_{\text {odd }}(x)-\left.\kappa f_{\text {odd }}(-x)\right|_{x=0}=\left.(1+\kappa) f_{\text {odd }}(x)\right|_{x=0},
\end{gathered}
$$

so that $f_{\text {even }}(0)$ exists, and equals $L_{\kappa} /(1-\kappa)$ and, because $\kappa \neq-1, f_{\text {odd }}(0)$ exists. Hence $f(0)=f_{\text {even }}(0)+f_{\text {odd }}(0)$ exists, that is, $f \in X$. When $\kappa=-1$, on the other hand, $f \in X_{-1}$ means that $f_{\text {even }}(0)$ exists since

$$
f_{\text {even }}=\frac{1}{2}(f+S f)=\frac{1}{2} f_{-1} .
$$

In this case

$$
X_{\text {ext }}=X_{\text {ext }}^{\{1\}}=X_{-1},
$$

and the extended point value is given as the symmetric distributional value

$$
T_{\text {ext }}(f)=T_{\text {ext }}^{\{1\}}(f)=f_{\text {even }}(0),
$$

or

$$
f(0)=f_{\text {even }}(0) \quad(\text { ext, } S) .
$$




\section{Dilations}

Let us keep working with $Y, X$, and $T$ as in (6), (7), and (8). Let $c>0$ be fixed and let us consider the operator

$$
H_{c}(f)(x)=f(c x) .
$$

$T$ is invariant with respect to $H_{c}$ and $X$.

In this case the spaces $X_{\kappa}, \kappa \neq 1$, are all different, and in fact $X_{\kappa_{1}, \ldots, \kappa_{n}}=X_{\lambda_{1}, \ldots, \lambda_{m}}$ if and only if $n=m$ and $\lambda_{1}, \ldots, \lambda_{n}$ is a permutation of $\kappa_{1}, \ldots, \kappa_{n}$.

In some cases the extended point value $T_{\text {ext }}(f)$ corresponds to a finite part at $x=0$ but that is not always the case. Indeed, suppose we have an asymptotic expansion of the form

$$
f(\varepsilon x)=\frac{a_{-\alpha_{n}}(x)}{\varepsilon^{\alpha_{n}}}+\cdots+\frac{a_{-\alpha_{1}}(x)}{\varepsilon^{\alpha_{1}}}+L+o(1),
$$

as $\varepsilon \rightarrow 0$ distributionally, where $0<\alpha_{1}<\cdots<\alpha_{n}$ and where the $a_{-\alpha_{j}}(x)$ are homogeneous distributions of degree $-\alpha_{j}$. Let $p$ be a polynomial of degree $n$ with $p(0)=1$ and $p(1) \neq 0$,

$$
p(x)=1+\sum_{j=1}^{n} A_{j} x^{j} .
$$

Then $p\left(H_{c}\right)=I+\sum_{j=1}^{n} A_{j} H_{c^{j}}$ so that the asymptotic expansion of $\left[p\left(H_{c}\right) f\right](\varepsilon x)$ as $\varepsilon \rightarrow 0$ is

$$
\left[p\left(H_{c}\right) f\right](\varepsilon x)=\frac{b_{-\alpha_{n}}(x)}{\varepsilon^{\alpha_{n}}}+\cdots+\frac{b_{-\alpha_{1}}(x)}{\varepsilon^{\alpha_{1}}}+p(1) L+o(1),
$$

where

$$
b_{-\alpha_{j}}(x)=p\left(c^{-\alpha_{j}}\right) a_{-\alpha_{j}}(x), \quad 1 \leq j \leq n
$$

Therefore, if we take

$$
p(x)=\prod_{j=1}^{n}\left(1-c^{\alpha_{j}} x\right),
$$

we obtain

$$
f_{c^{\alpha_{1}}, \ldots, c^{\alpha_{1}}}(\varepsilon x)=\left[p\left(H_{c}\right) f\right](\varepsilon x)=p(1) L+o(1), \quad \text { as } \varepsilon \rightarrow 0,
$$

so that $f_{c^{\alpha_{1}}, \ldots, c^{\alpha_{1}}} \in X$ and (5) yields

$$
T_{\text {ext }}(f)=\frac{T\left(f_{c^{\alpha_{1}}, \ldots, c^{\alpha_{1}}}\right)}{p(1)}=L
$$

that is

$$
f(0)=L \quad\left(\text { ext }, H_{c}\right) .
$$

In other words, $T_{\text {ext }}(f)$ is the finite part of the limit of $f(\varepsilon x)$ as $\varepsilon \rightarrow 0$.

Consider next the distribution

$$
g(x)=|x|^{i}=\sin \ln |x|+i \cos \ln |x| .
$$

The distributional point value $g(0)$ does not exist [6]. The function $g$ does not have an asymptotic expansion of the form (9) either. Here we have that

$$
H_{c}(g)(x)=c^{i} f(x) .
$$

If $c \neq e^{2 n \pi}, n \in \mathbb{Z}$, then $g_{c^{-i}}=g-c^{-i} H_{c}(g)$ belongs to $X$ since it actually vanishes identically, and consequently

$$
\begin{gathered}
T_{\text {ext }}(g)=\frac{1}{1-c^{-i}} T\left(g_{c^{-i}}\right)=0, \\
g(0)=0 \quad\left(\text { ext }, H_{c}\right) .
\end{gathered}
$$

Interestingly, the point value in the sense of Campos Ferreira $g(0)$ exists and equals zero, $g(0)=0(\mathrm{CF})[6]$. 


\section{Extended Cesàro summability}

We may employ several different notions of point values to obtain corresponding notions of summability of series. In particular if we use Fourier series we may consider summability of a series $\sum_{n=-\infty}^{\infty} a_{n}$ by studying the distributional point value of the generalized function

$$
f(x)=\sum_{n=-\infty}^{\infty} a_{n} e^{i n x},
$$

at $x=0$. The characterization of the point values of periodic distributions was first given in [2], and it can be found in [4, Chapter 6]. Indeed, using the notion of Cesàro summability, denoted as $(\mathrm{C}, k)$, we have the ensuing result.

Theorem 7.1. Let $f \in \mathcal{S}^{\prime}$ be a periodic distribution of period $2 \pi$ and let $\sum_{n=-\infty}^{\infty} a_{n} e^{i n x}$ be its Fourier series. Let $x_{0} \in \mathbb{R}$. Then

$$
f\left(x_{0}\right)=\gamma, \quad \text { in } \mathcal{D}^{\prime}
$$

if and only if there exists $k$ such that

$$
\lim _{x \rightarrow \infty} \sum_{-x \leq n \leq a x} a_{n} e^{i n x_{0}}=\gamma
$$

for each $a>0$.

This theorem gives the complete characterization of point values. There are several important particular cases, as we now explain. When $f$ is symmetric about $x=x_{0}$, i.e., $f\left(x-x_{0}\right)=f\left(x_{0}-x\right)$, then $f\left(x_{0}\right)=\gamma$ in $\mathcal{D}^{\prime}$ if and only if $\sum_{n=-\infty}^{\infty} a_{n} e^{i n x_{0}}=\gamma \quad(\mathrm{C})$. Also, if the Fourier series of $f$ is of the power series type, i.e., $f(x)=\sum_{n=0}^{\infty} a_{n} e^{i n x}$, then $f\left(x_{0}\right)=\gamma$ in $\mathcal{D}^{\prime}$ if and only if $\sum_{n=0}^{\infty} a_{n} e^{i n x_{0}}=\gamma \quad(\mathrm{C})$.

Let us thus consider the following spaces and operators

$$
\widetilde{Y}=\left\{\left\{a_{n}\right\}_{n=0}^{\infty}: \sum_{n=0}^{\infty} a_{n} e^{i n x} \in \mathcal{D}^{\prime}(\mathbb{R})\right\},
$$

in other words [4,9], $\left\{a_{n}\right\}_{n=0}^{\infty} \in \widetilde{Y}$ if and only if there exists $\alpha>0$ such that $\left|a_{n}\right|=O\left(n^{\alpha}\right)$ as $n \rightarrow \infty$. The space $\widetilde{X}$ is defined as

$$
\widetilde{X}=\left\{\left\{a_{n}\right\}_{n=0}^{\infty} \in \tilde{Y}: \sum_{n=0}^{\infty} a_{n} \text { is Cesàro summable }\right\} .
$$

The results from [2] and [4, Chapter 6] that we quoted yield that $\left\{a_{n}\right\}_{n=0}^{\infty} \in \widetilde{X}$ if and only if the distribution

$$
f(x)=\mathcal{F}\left\{\left\{a_{n}\right\}_{n=0}^{\infty} ; x\right\}=\sum_{n=0}^{\infty} a_{n} e^{i n x},
$$

has a distributional point value at 0 . The operator $\widetilde{T}$ in this case is given as the Cesàro sum

$$
\widetilde{T}\left(\left\{a_{n}\right\}\right)=\sum_{n=0}^{\infty} a_{n} \quad(\mathbf{C}) .
$$

We also have

$$
\widetilde{T}\left(\left\{a_{n}\right\}\right)=f(0),
$$

where $f(0)$ is the distributional point value.

If $q$ is an integer, $q \geq 2$, we can consider the operator $\widetilde{H}_{q}$ given as $\widetilde{H}_{q}\left(\left\{a_{n}\right\}\right)=\left\{b_{n}\right\}$ where

$$
b_{q n}=a_{n}, \quad b_{q n+j}=0, \quad 1 \leq j \leq q-1 .
$$

In this case $\widetilde{H}_{q}$ is not an isomorphism of $\widetilde{X}$, but $\widetilde{T}$ is "almost" invariant with respect to $\widetilde{H}_{q}$ and $\widetilde{X}$. In fact, in the correspondence $\left\{a_{n}\right\} \longleftrightarrow f$ then $\widetilde{H}_{q}\left\{a_{n}\right\}$ corresponds to the operator $H_{q}(f)$ considered in the Section 6 ,

$$
\mathcal{F}\left\{\left\{b_{n}\right\}_{n=0}^{\infty} ; x\right\}=\sum_{n=0}^{\infty} b_{n} e^{i n x}=\sum_{m=0}^{\infty} a_{m} e^{i q m x}=\mathcal{F}\left\{\left\{a_{n}\right\}_{n=0}^{\infty} ; q x\right\}
$$

that is

$$
\mathcal{F}\left\{\widetilde{H}_{q}\left\{a_{n}\right\} ; x\right\}=H_{q}(f)(x) .
$$


It would be possible to consider an extension procedure for $\widetilde{T}$ with respect to $\widetilde{H}_{q}$, but it is simpler to define

$$
\sum_{n=0}^{\infty} a_{n}=\gamma \quad\left(\text { ext, } \widetilde{H}_{q}\right)
$$

if

$$
f(0)=\gamma \quad\left(\text { ext, } H_{q}\right)
$$

\section{An example}

Let us consider the sequence $\left\{n^{s}\right\}_{n=1}^{\infty}$ for $s \in \mathbb{C}$. The series $\sum_{n=1}^{\infty} n^{s}$ converges for $\Re e s<-1$ and of course when this holds

$$
\sum_{n=1}^{\infty} n^{s}=\zeta(-s)
$$

where $\zeta$ is the Riemann zeta function. Our aim is to find an extended value of the series, $\sum_{n=1}^{\infty} n^{s}$ (ext, $\left.\widetilde{H}_{q}\right)$ when the series diverges, that is, when $\Re e s \geq-1$.

Let us define the distribution

$$
f(x)=\sum_{n=1}^{\infty} n^{s} e^{i n x}
$$

convergence in $\mathcal{D}^{\prime}(\mathbb{R})$. The distributional point value $f(0)$ exists and equals $\zeta(-s)$ when $\Re e s<-1$ but it does not exist otherwise. In fact, the behavior of $f(\varepsilon x)$ as $\varepsilon \rightarrow 0$ is as follows.

Lemma 8.1. If $\Re e s \geq-1, s \neq-1$, then

$$
f(\varepsilon x)=\frac{C_{s}}{\varepsilon^{s+1}}(x+i 0)^{-s-1}+\zeta(-s)+o(\varepsilon),
$$

distributionally, where

$$
C_{s}=e^{i \pi(s+1) / 2} \Gamma(s+1),
$$

and where $(x+i 0)^{\alpha}$ is the distribution $\lim _{y \rightarrow 0^{+}}(x+i y)^{\alpha}$ if $\alpha \in \mathbb{C}$.

Proof. Let $\phi \in \mathcal{D}(\mathbb{R})$. Then

$$
\langle f(\varepsilon x), \phi(x)\rangle=\sum_{n=1}^{\infty} n^{s}\left\langle e^{i n \varepsilon x}, \phi(x)\right\rangle=\sum_{n=1}^{\infty} n^{s} \widehat{\phi}(n \varepsilon),
$$

where $\widehat{\phi}(u)=\int_{-\infty}^{\infty} e^{i u x} \phi(x) \mathrm{d} x$ is the Fourier transform of $\phi$. The asymptotic expansion of this series can be obtained from an asymptotic formula of Ramanujan [4, (5.15)], namely,

$$
\sum_{n=1}^{\infty} n^{s} \psi(n \varepsilon) \sim \frac{1}{\varepsilon^{s+1}} \int_{0}^{\infty} x^{s} \psi(x) \mathrm{d} x+\sum_{j=0}^{\infty} \zeta(-s-j) \frac{\psi^{(j)}(0)}{j !} \varepsilon^{j},
$$

as $\varepsilon \rightarrow 0$ if $\psi$ is smooth in $\mathbb{R}$ and of exponential decrease at infinity. If $\psi=\widehat{\phi}$ we obtain

$$
\begin{aligned}
\sum_{n=1}^{\infty} n^{s} \widehat{\phi}(n \varepsilon) & =\frac{1}{\varepsilon^{s+1}}\left\langle u_{+}^{s}, \widehat{\phi}(u)\right\rangle+\zeta(-s)+o(\varepsilon) \\
& =\frac{1}{\varepsilon^{s+1}}\left\langle\widehat{u_{+}^{s}}(x), \phi(x)\right\rangle+\zeta(-s)+o(\varepsilon) \\
& =\frac{C_{s}}{\varepsilon^{s+1}}\left\langle(x+i 0)^{-s-1}, \phi(x)\right\rangle+\zeta(-s)+o(\varepsilon)
\end{aligned}
$$

since [9] the Fourier transform of $u_{+}^{s}$ is precisely $C_{s}(x+i 0)^{-s-1}$.

Applying the extension procedure corresponding to the operator $H_{c}$, for any $c>0$, the analysis of the Section 6 yields that the extended point value of $f$ at 0 is the finite part of the expansion (10), hence

$$
f(0)=\zeta(-s), \quad\left(\text { ext, } H_{c}\right),
$$

for $\Re e s \geq-1, s \neq-1$. In fact, (11) also holds when $\Re e s<-1$ as a distributional point value, no extension needed. 
Using the extended sum operator of the previous section we obtain

$$
\sum_{n=1}^{\infty} n^{s}=\zeta(-s), \quad\left(\text { ext, } \widetilde{H}_{q}\right), \quad s \neq-1 .
$$

We can also derive formula (12) by using the method employed by Sasane [15], to find an extended sum for the divergent series $\sum_{n=1}^{\infty} n$. Sasane was inspired by a trick used by Ramanujan, but the method is probably much older. Indeed, using the operator $\mathrm{H}_{2}$,

$$
f_{\kappa}(x)=f(x)-\kappa f(2 x)=\sum_{n=1}^{\infty} n^{s} e^{i n x}-\kappa \sum_{n=1}^{\infty} n^{s} e^{2 i n x},
$$

so that if $\kappa=2^{s+1}$,

$$
f_{2^{s+1}}(x)=\sum_{n=1}^{\infty}(-1)^{n+1} n^{s} e^{i n x} .
$$

Notice now that the series $\sum_{n=1}^{\infty}(-1)^{n+1} n^{s}$ is always Cesàro summable [4, pg. 251], that is, for all $s \in \mathbb{C}$

$$
\sum_{n=1}^{\infty}(-1)^{n+1} n^{s}=\left(1-2^{s+1}\right) \zeta(-s) \quad(\mathbf{C}) .
$$

Therefore the point value $f_{2^{s+1}}(0)$ exists for any $s$ and

$$
f_{2^{s+1}}(0)=\left(1-2^{s+1}\right) \zeta(-s) .
$$

Hence if $s \neq-1$, namely, if $\kappa \neq 1$,

$$
T_{\text {ext }}(f)=\frac{f_{2^{s+1}}(0)}{1-2^{s+1}}=\zeta(-s),
$$

and consequently,

$$
\sum_{n=1}^{\infty} n^{s}=\zeta(-s), \quad\left(\text { ext, } \widetilde{H}_{2}\right)
$$

\section{An example in several variables}

We may consider the following spaces and operators

$$
\begin{gathered}
Y=\mathcal{D}^{\prime}\left(\mathbb{R}^{n}\right), \\
X=\{f \in Y: \text { the distributional point value } f(\mathbf{0}) \text { exists }\},
\end{gathered}
$$

and

$$
T(f)=f(\mathbf{0}) .
$$

Let $A$ be an invertible $n \times n$ matrix and let us define $S_{A}$ to be the operator

$$
S_{A}(f)(\mathbf{x})=f(A \mathbf{x}) .
$$

Clearly $T$ is invariant with respect to $S_{A}$ and $X$. The extension procedure is applicable.

To illustrate our ideas, let us consider a particular case. We take $n=2$ and $A$ the counterclockwise rotation of angle $2 \pi / 3$, so that $A^{3}=I$. As we shall see, in this case $X_{\text {ext }} \neq X_{\text {ext }}^{\{1\}}$, but

$$
X_{\mathrm{ext}}=X_{\mathrm{ext}}^{\{2\}}, \quad T_{\mathrm{ext}}=T_{\mathrm{ext}}^{\{2\}} .
$$

Indeed, $X_{\kappa} \neq X$ for just two values of $\kappa$, namely $\omega=e^{2 \pi i / 3}$ and $\omega^{2}$. This can be seen by writing

$$
f=f_{1}+f_{2}+f_{3},
$$

where

$$
\begin{gathered}
f_{1}=\frac{f+S_{A}(f)+S_{A^{2}}(f)}{3}, \\
f_{2}=\frac{f+\omega^{2} S_{A}(f)+\omega S_{A^{2}}(f)}{3},
\end{gathered}
$$

and

$$
f_{3}=\frac{f+\omega S_{A}(f)+\omega^{2} S_{A^{2}}(f)}{3} .
$$


Notice that

$$
S_{A}\left(f_{1}\right)=f_{1}, \quad S_{A}\left(f_{2}\right)=\omega f_{2}, \quad S_{A}\left(f_{3}\right)=\omega^{2} f_{2} .
$$

Hence if $f-\kappa S_{A}(f) \in X$ then

$$
(1-\kappa) f_{1}+(1-\kappa \omega) f_{2}+\left(1-\kappa \omega^{2}\right) f_{3} \in X
$$

and since $S_{A}(X)=X$,

$$
(1-\kappa) f_{1}+(1-\kappa \omega) \omega f_{2}+\left(1-\kappa \omega^{2}\right) \omega^{2} f_{3} \in X,
$$

and also, because $\omega^{4}=\omega$,

$$
(1-\kappa) f_{1}+(1-\kappa \omega) \omega^{2} f_{2}+\left(1-\kappa \omega^{2}\right) \omega f_{3} \in X .
$$

Solving the corresponding system of equations yields

$$
(1-\kappa) f_{1} \in X, \quad(1-\kappa \omega) f_{2} \in X, \quad\left(1-\kappa \omega^{2}\right) f_{3} \in X .
$$

If $\kappa \neq 1, \omega$, and $\omega^{2}$ then it follows that $f_{1}, f_{2}$, and $f_{3}$ all belong to $X$ and thus so does $f$. Similarly we obtain

$$
\begin{gathered}
X_{\omega}=\left\{f: f_{1}, f_{2} \in X\right\}, \\
X_{\omega^{2}}=\left\{f: f_{1}, f_{3} \in X\right\} .
\end{gathered}
$$

Iteration of these results gives

$$
X_{\omega, \omega}=X_{\omega}, \quad X_{\omega^{2}, \omega^{2}}=X_{\omega^{2}},
$$

and

$$
X_{\omega, \omega^{2}}=X_{\omega^{2}, \omega}=\left\{f: f_{1} \in X\right\} .
$$

Therefore

$$
X_{\mathrm{ext}}=X_{\mathrm{ext}}^{\{2\}}=\left\{f: f_{1} \in X\right\},
$$

and

$$
T_{\text {ext }}(f)=T_{\text {ext }}^{\{2\}}(f)=T\left(f_{1}\right),
$$

that is

$$
f(\mathbf{0})=f_{1}(\mathbf{0}) \quad\left(\text { ext, } S_{A}\right)
$$

\section{References}

[1] T. J. Bromwich, An Introduction to the Theory of Infinite Series, Third Edition, Chelsea, New York, 1991.

[2] R. Estrada, Characterization of the Fourier series of distributions having a value at a point, Proc. Amer. Math. Soc. 124 (1996) 1205-1212

[3] R. Estrada, Evaluation of convergent series by using finite parts, Open J. Math. Sci. 4 (2020) 98-109.

[4] R. Estrada, R. P. Kanwal, A Distributional Approach to Asymptotics. Theory and Applications, Second Edition, Birkhäuser, Boston, 2002.

[5] R. Estrada, J. Vindas, A general integral, Disertationes Math. (Rozprawy Mat.) 483 (2012) 1-49.

[6] J. C. Ferreira, Introduction to the Theory of Distributions, Longman, London, 1997.

[7] J. Hadamard, Lectures on Cauchy's Problem in Linear Differential Equations, Dover, New York, 1952.

[8] G. H. Hardy, Divergent Series, Clarendon Press, Cambridge, 1949.

[9] R. P. Kanwal, Generalized Functions: Theory and Technique, Third Edition, Birkhäuser, Boston, 2004.

[10] S. Łojasiewicz, Sur la valeur et la limite d'une distribution en un point, Studia Math. 16 (1957) 1-36.

[11] S. Łojasiewicz, Sur la fixation de variables dans une distribution, Studia Math. 17 (1958) 1-64.

[12] S. Paycha, Regularised Integrals, Sums and Traces: An Analytic Point of View, Amer. Math. Soc., Providence, 2012.

[13] S. Pilipović, B. Stanković, A. Takači, Asymptotic Behavior and Stieltjes Transformation of Distributions, Teubner-Texte zur Mathmatik, Leipzig, 1990.

[14] S. Pilipović, B. Stanković, J. Vindas, Asymptotic Behavior of Generalized Functions, World Scientific, Singapore, 2011.

[15] A. Sasane, A summation method based on the Fourier series of periodic distributions and an example arising in the Casimir effect, Indag. Math. 31 (2020) 477-504.

[16] L. Schwartz, Thèorie des Distributions, Second Edition, Hermann, Paris, 1966.

[17] V. S. Vladimirov, Y. N. Drozhzhinov, B. I. Zavialov, Tauberian theorems for generalized functions, Kluwer Academic, Dordrecht, 1988. 